\title{
AKAP signaling complexes: getting to the heart of the matter
}

\author{
George McConnachie, Lorene K. Langeberg and John D. Scott
}

Howard Hughes Medical Institute, Vollum Institute, L-474, Oregon Health \& Science University, 3181 S.W. Sam Jackson Park Road, Portland, OR 97239, USA

Subcellular compartmentalization of protein kinases and phosphatases through their interaction with A-kinase anchoring proteins (AKAPs) provides a mechanism to control signal transduction events at specific sites within the cell. Recent findings suggest that these anchoring proteins dynamically assemble different cAMP effectors to control the cellular actions of cAMP spatially and temporally. In the heart, signaling events such as the onset of cardiac hypertrophy are influenced by muscle-specific mAKAP signaling complexes that target protein kinase $A$ (PKA), the cAMP-responsive guanine-nucleotide exchange factor EPAC and cAMP-selective phosphodiesterase 4 (PDE4). Mediation of signaling events by AKAPs might also have a role in the control of lipolysis in adipocytes, where insulin treatment reduces the association of AKAPs with G-protein-coupled receptors. These are only two examples of how AKAPs contribute to specificity in cAMP signaling. This review will explore recent development that illustrates the role of multiprotein complexes in the regulation of CAMP signaling.

\section{A-kinase anchoring proteins: focal points in cAMP signaling}

Binding of extracellular messengers to receptors on the cell surface initiates transmission of a signal via synthesis of second messengers (see Glossary) [1]. Small molecules such as cAMP are rapidly formed and diffuse into the cytosol to activate downstream effector proteins such as cyclic-nucleotide-gated (CNG) channels, guanine-nucleotide exchange proteins (EPACs) and the serine-threonine protein kinase A (PKA). This process provides a mechanism whereby extracellular stimuli can influence diverse biological processes. Conversely, signal termination is achieved because members of the phosphodiesterase (PDE) superfamily locally degrade cAMP into $5^{\prime}$ adenosine monophosphate (5'-AMP). A-kinase anchoring proteins (AKAPs) contribute to the specificity of cAMP signaling by targeting PKA in proximity to cAMP gradients generated by the counterbalancing activities of adenylyl cyclases and PDEs. This provides a mechanism by which cAMP regulates cellular responses to external stimuli within specific subcellular regions and for limited time intervals $[2,3]$.

Corresponding author: Scott, J.D. (scott@ohsu.edu).
Here, we will focus on recent findings on the role of AKAPs in the control of cAMP gradients, which are important in widespread biological phenomena. An example of this is the assembly of a multienzyme complex by the muscle-specific mAKAP to co-ordinate cAMPeffector regulation of signaling pathways involved in cardiac hypertrophy. Similarly, it has been shown that AKAPs are involved in insulin action in adipose tissue [4], although the AKAP that is responsible for targeting PKA to G-protein-coupled receptors in adipocytes has not been yet identified.

\section{Dynamic cAMP gradients}

To date, there are nine known members of the mammalian adenylyl cyclase family that synthesize cAMP from ATP in response to G-protein-coupled-receptor stimulation $[5,6]$ (Figure 1). The ability of different hormones to induce diverse cellular responses through the same second messenger suggests that there are mechanisms in place to differentiate cAMP signals. Specificity is, in part, provided by selective tissue distribution and differential modes of regulation. Although experiments that use radiometric and immunoassays can only detect total cellular cAMP at one time point, modified cAMP effectors

\footnotetext{
Glossary

Activity reporter: a construct that detects enzyme activity in cells.

Adenylyl cyclase: a membrane-bound enzyme that generates cAMP in response to receptor stimulation.

Adipocytes: cells from fat tissue that are specialized in storing energy as fat. cAMP: cyclic adenosine $3^{\prime}, 5^{\prime}$-monophosphate is generated from ATP by adenylyl cyclase in response to the activation of many types of cell receptors. Exchange protein directly activated by cAMP (EPAC): guanine-nucleotide exchange factor activated by cAMP.

Fluorescence energy transfer (FRET): a technique to measure the relative distances of two fluorescent molecules in cells.

G-protein-coupled receptor: a cell-surface receptor that binds extracellular ligands such as hormones or neurotransmitters and transmits the signal through heterotrimeric G-proteins (GTP binding, membrane-bound proteins) to mediate various signal transducing systems.

Hypertrophy: an enlargement of an organ in the body caused by an increase in cell size.

Lipolysis: the process by which lipids are hydrolyzed.

Multi-enzyme complex: a complex of signaling proteins that functions to regulate the biological outcome of an upstream signal.

Phosphodiesterase (PDE): it hydrolyses cAMP and/or cGMP to AMP and GMP, respectively.

Protein kinase A (PKA): serine-threonine kinase that is activated by cAMP. It relays a signal by phosphorylating downstream substrate targets.

Second messengers: small molecules that are generated or released in the cytosol to relay a signal to the interior of the cell in reponse to an extracellular signal (e.g. cAMP).

Ventriculocytes: primary muscle cells derived from heart tissue.
} 


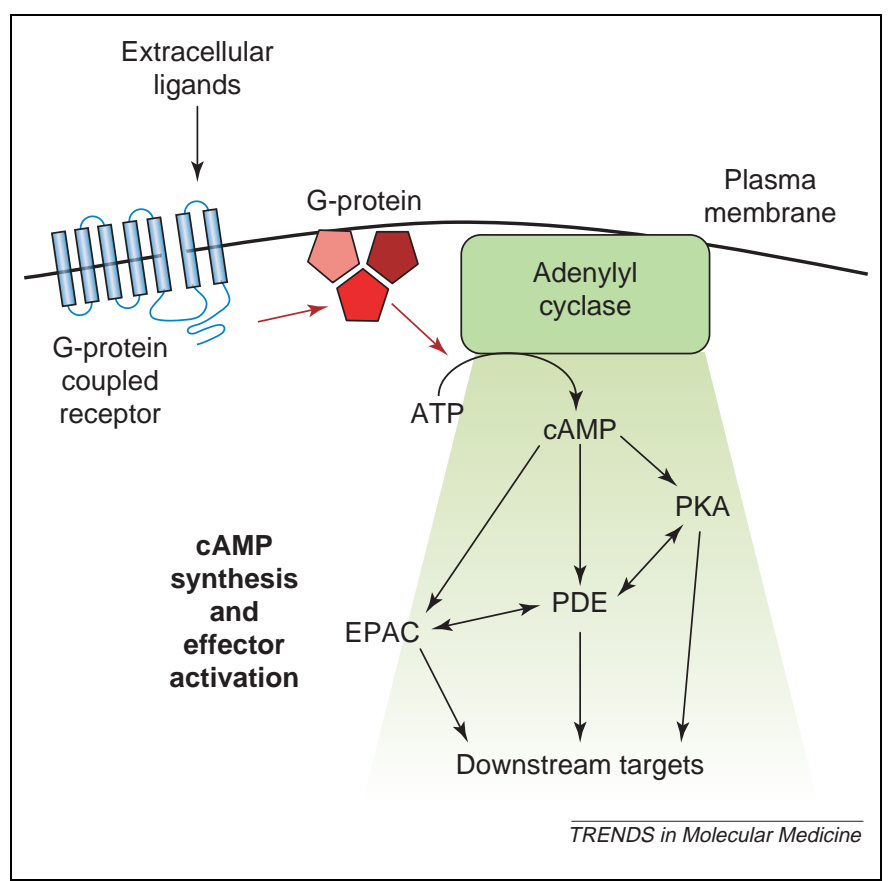

Figure 1. Schematic diagram of cAMP synthesis and downstream effector activation. When an extracellular ligand such as an hormone binds to and activates a seven-transmembrane G-protein-coupled receptor, the signal is passed through the heterotrimeric $\mathrm{G}$ protein to adenylyl cyclase. The activated adenylyl cyclase converts ATP into the second messenger cAMP. As the gradient of cAMP concentration diffuses in the cell, various enzymes or effectors are activated. These include PKA, PDE and EPAC. The interplay of each of these effectors as they interact directly or indirectly with each other and other downstream targets is currently an area of intense study.

are now being used to investigate the temporal and spatial aspects of cAMP signaling in living cells [4,7-15]. Although the diffusion of cAMP has long been considered to be fast within cells, recent studies have suggested that enzymes involved in cAMP synthesis and degradation generate boundaries for cAMP diffusion [7-9,16,17].

Studies in HEK293 cells where rat olfactory CNG channels, which have been modified to be sensitive to cAMP, were overexpressed have illustrated that distinct cAMP signals coexist within these cells [10]. In these studies, local changes in cAMP levels were determined by fluorescent monitoring of $\mathrm{Ca}^{2+}$ influx through mutant CNG channels, whereas global cAMP changes were determined by measuring the conversion of $\left[{ }^{3} \mathrm{H}\right] \mathrm{ATP}$ into $\left[{ }^{3} \mathrm{H}\right] \mathrm{cAMP}$. Using these two methods to measure cAMP gradients, Rich et al. [10] observed that upon prostaglandin-E1 stimulation there was a transient increase of cAMP level in proximity to the membrane, whereas total cellular cAMP rose to a sustained level. Another approach to investigate the spatial and temporal control of cAMP gradients in living cells used fluorescent resonance energy transfer (FRET) reporters with fluorescently labeled regulatory and catalytic subunits of PKA [11,12]. These reporters have been successfully used to demonstrate that stimulation of $\beta$-adrenergic receptors in neonatal rat cardiac myocytes results in the generation of cAMP gradients [13].

Conversely, termination of cyclic-nucleotide signaling is achieved by PDEs, a superfamily of $>70$ different isozymes that degrade cAMP and cyclic GMP (cGMP). Distinctive tissue distribution, subcellular compartmentalization and differential regulation of these enzymes contribute to the establishment of local cAMP gradients by limiting the diffusion of cAMP that is generated by adenylyl cyclases $[18,19]$. The type- 4 PDEs are a family of $>16$ distinct isoforms that have a conserved catalytic core. Divergence within the $\mathrm{N}$-terminal region of PDE4 isoforms enables association with various proteins and, therefore, differential subcellular targeting and regulation [19]. Importantly, the PDE4D3 isozyme has been shown to be part of signaling complexes that target PKA. These two cAMPresponsive enzymes (PDE and PKA) are found in complex with a growing number of AKAPs [20,21], thereby enabling tight regulation of cAMP.

\section{AKAPs: a family of proteins that target PKA}

AKAPs are a family of $>50$ anchoring proteins that, although being structurally diverse, have in common the ability to bind to and target PKA [2,3] (Figure 2). PKA is a heterotetramer that consists of two catalytic (C) subunits held in an inactive conformation by a regulatory (R) subunit dimer. The type-I PKA holoenzyme contains RI subunits (RI $\alpha$ or $\mathrm{RI} \beta$ ) and is primarily cytoplasmic, whereas the type-II holoenzyme contains RII subunits (RII $\alpha$ or RII $\beta$ ) and is associated with particulate subcellular fractions. Binding of cAMP to PKA R subunits releases the active $\mathrm{C}$ subunit $(\mathrm{C} \alpha, \mathrm{C} \beta$ or $\mathrm{C} \gamma)$ to phosphorylate nearby substrates [22]. Anchoring of PKA to an AKAP is achieved by the interaction of the R-subunit dimer with a $14-18$ amino acid amphipathic $\alpha$-helix region of AKAP [22-24]. Disruption PKA anchoring has been demonstrated experimentally using Ht31, a peptide that encompasses this amphipathic $\alpha$ helical region from an AKAP, AKAP-Lbc, which has a high affinity for the RII

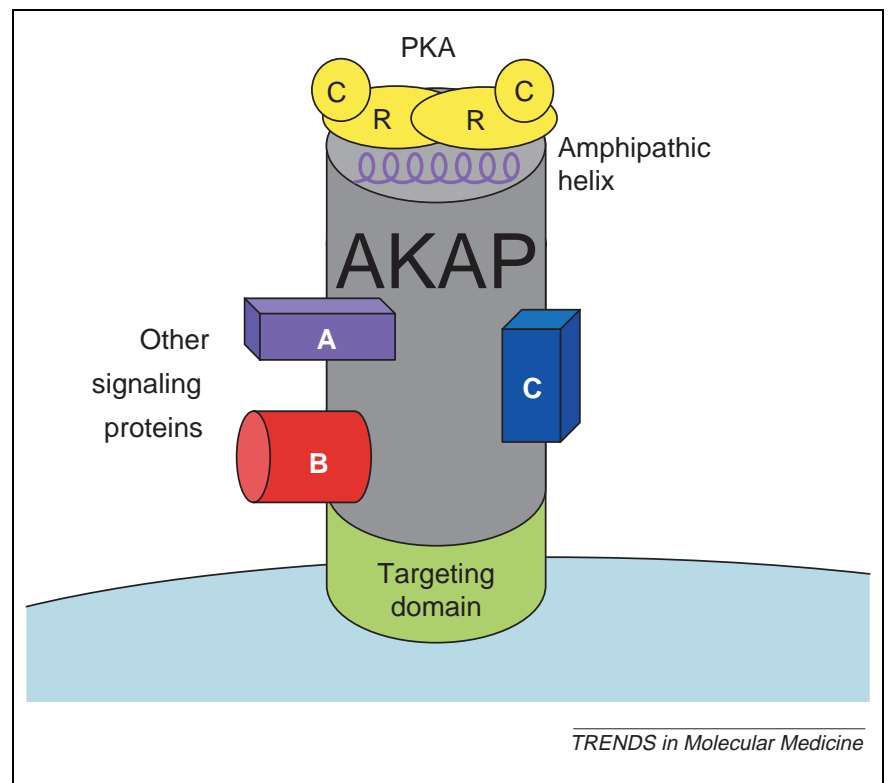

Figure 2. Schematic diagram of a prototypic AKAP. Anchoring of PKA (yellow) to AKAP is accomplished through hydrophobic interaction between the amphipathic $\alpha$ helical region of AKAP and the surface formed by the $\mathrm{N}$-terminal dimerization region of the two $R$ subunits of PKA. When cAMP binds to the $R$ subunit, the $C$ subunit of PKA is activated and released to phosphorylate nearby substrates. AKAP also serves as a signaling scaffold for various other signaling enzymes ( $A, B$ and $C)$. Finally, the targeting region of AKAP (green) localizes the entire complex to the appropriate subcellular compartment via protein-protein or protein-lipid interactions. 
Table 1. Summary of a selection of AKAP complexes

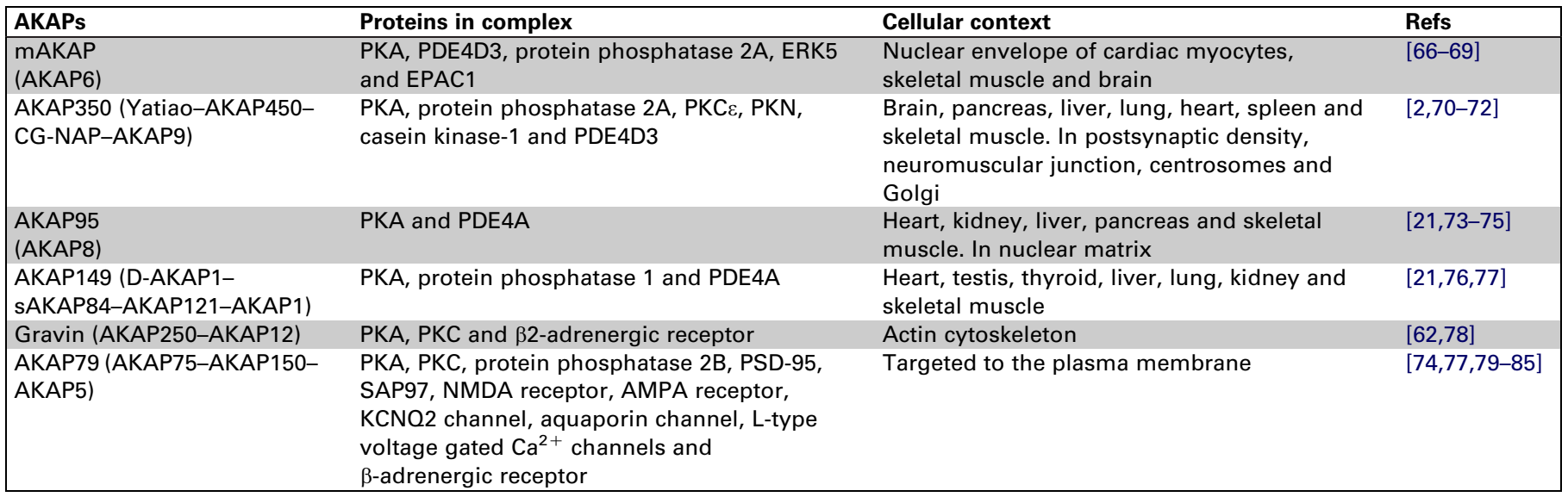

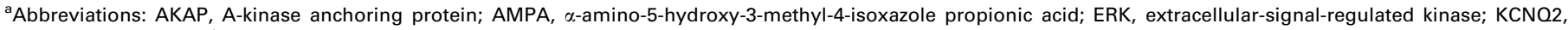

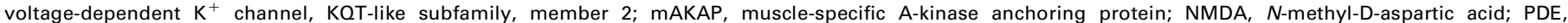
phosphodiesterase; PKA, protein kinase A; PKC $\varepsilon$, protein kinase C\&; PKN, protein kinase N; PSD-95, postsynaptic protein of $95 \mathrm{kD}$; SAP97, synapse-associated protein 97.

subunits of PKA. Although most AKAPs that have been characterized bind to RII subunits with high affinity, several AKAPs have been reported to interact specifically with RI [25]. D-AKAP1 and D-AKAP2 are examples of dual-specificity AKAPs that can anchor both types of $R$ subunit [26-28]. AKAPs also have unique protein-lipid or protein-protein targeting domains that tether the PKA-AKAP complex to distinct subcellular locations to respond to local cAMP gradients $[3,22,29,30]$. In support of this, Zhang et al. [14] used a genetically encoded PKAactivity reporter - A-kinase activity reporter (AKAR) and demonstrated that, when PKA was tethered in the same complex as its substrate, the rate of phosphorylation was enhanced 3-4-fold. This provided evidence that, in response to cAMP, the release of $\mathrm{C}$ subunit from an AKAP complex would preferentially phosphorylate a local pool of substrate.

\section{AKAP signaling complexes: mAKAPs}

An important feature of AKAPs is their ability to interact with other signaling proteins in addition to PKA (Table 1). These multienzyme complexes contain signal-termination enzymes, such as phosphatases and PDEs, and signaltransduction enzymes, such as kinases. Therefore, these enzymes can both upregulate and downregulate specific signaling pathways [3]. Currently, the focus of AKAP research is the identification of proteins that are associated with AKAPs. This provides candidate protein substrates for the anchored PKA. It also enables the study of how the interplay among AKAP-associated proteins can contribute to downstream biological response. Using this approach, it is becoming clear how enzymes of the mAKAP complex (Figure 3) are involved in the regulation cardiac function.

Immunoprecipitation studies from rat heart extracts demonstrated that PDE4D co-purified with both the RII subunit of PKA and mAKAP [20]. Additionally, it has been shown that the PDE4D3 isoform directly interacts with mAKAP and that it co-localizes with mAKAP at the nuclear membrane of cardiomyocytes [20]. The finding that both PDE4D3 and PKA are associated with mAKAP raised the possibility that the complex tightly regulates local cAMP concentrations and mAKAP-associated PKA activity. Indeed, inhibition of mAKAP-associated PDE4 activity enhanced the activity of the anchored PKA [20]. It has also been observed that phosphorylation of PDE4 by PKA enhanced its PDE activity [19,31]. PDE4D3 is phosphorylated by PKA at two sites, Ser13 and Ser54. Phosphorylation at Ser54 results in a 2-3-fold enhancement in PDE activity [31]. Furthermore, experiments using the PKA inhibitor peptide (PKI) or a PKA-anchoring inhibitor peptide (Ht31) demonstrated that mAKAPassociated PKA phosphorylation caused a two-fold increase of PDE4D3 activity [20]. These observations suggested that mAKAP maintains a cAMP signaling complex in which PKA activity is attenuated by PDE

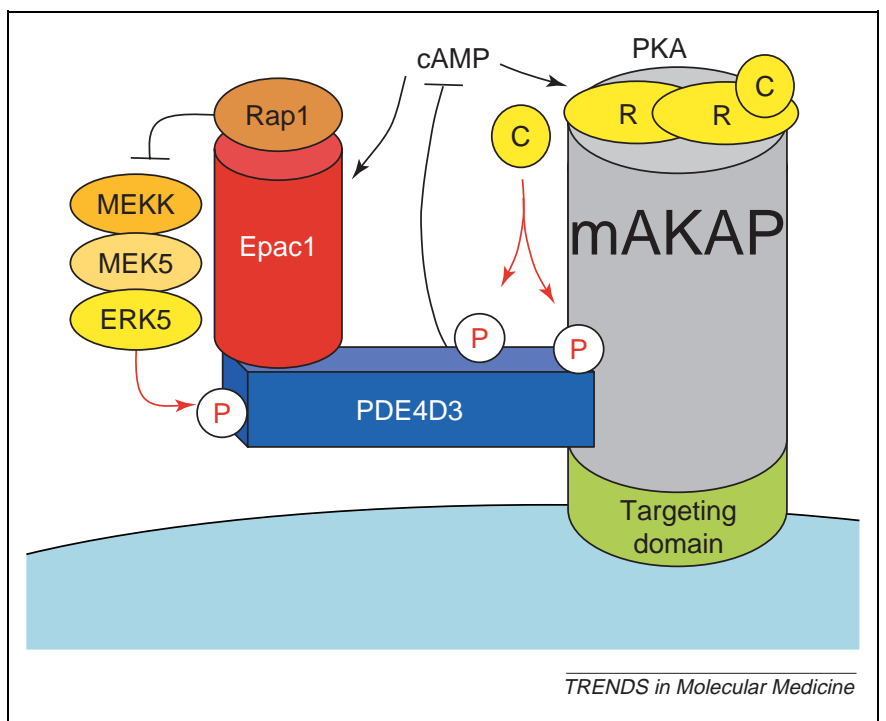

Figure 3. The mAKAP complex at the nuclear membrane of cardiomyocytes brings together three cAMP effectors: PKA, PDE4D3 and Epac1. Each of these enzymes is activated at different concentrations of cAMP and there is interaction between them. As cAMP levels rise, activated PKA phosphorylates PDE4D3 in the complex at two distinct sites (double red arrow). Phosphorylation of Ser13 enhances the PDE affinity for mAKAP and phosphorylation of Ser54 increases the activity of PDE. This increase in activity depletes the local concentrations of CAMP and enables reformation of the inactive PKA holoenzyme. As cAMP levels fall, the Epac1mediated inhibition of ERK5 pathway is blocked and the consequent ERK phosphorylation (red arrow) of PDE4D3 decreases the PDE activity, enabling accumulation of more cAMP. 
activity in a negative-feedback loop. Under basal conditions, mAKAP-associated PDE4D3 maintains local cAMP below the threshold level required to activate the anchored PKA. Upon hormonal stimulation, increased cAMP levels overcome the PDE activity, releasing active PKA C subunit from the AKAP complex. This leads to PKA phosphorylation of the tethered PDE4D3 on Ser54, thus increasing the local PDE activity. The subsequent increase in cAMP metabolism returns cAMP levels to basal, favouring the reformation of the PKA holoenzyme.

Although phosphorylation of Ser13 by PKA does not affect PDE activity, it enhances the affinity of PDE4D3 for mAKAP [32]. In addition, PKA phosphorylation has been shown to modulate protein-protein interactions within several other AKAP complexes [33,34]. In the case of mAKAP, functional consequences of this type of regulation might be that PKA phosphorylates PDE4D3 at Ser13 and Ser54 in response to hormonal stimulation. If this is the case, PDE is drawn into the mAKAP complex and has increased activity, thereby decreasing the local levels of cAMP back to basal levels. Using modified versions of the AKAR2-PKA reporter, Dodge-Kafka et al. [15] examined whether this arrangement of PKA and PDE would generate local fluctuations in cAMP and pulses of compartmentalized PKA activity. The AKAR2-PKA activity reporter is sensitive to PDE activity in addition to PKA activity, thereby monitoring both the activation and termination of PKA signaling [4]. The modified AKAR2 reporters, AKAR-PKA and AKAR-PKA-PDE, bind PKA alone or PKA and PDE4D3, respectively [15]. Upon cAMP stimulation, cells that express AKAR-PKA reporter exhibited sustained elevation of FRET, indicating that the PKA associated with AKAR2 was activated. However, in cells that express the AKAR-PKA-PDE reporter, the FRET response was transient, presumably due to a drop in cAMP levels because the associated PDE activity increased [15]. This reporter serves as a model for the recruitment of PKA and PDE into a single complex to control localized cAMP gradients.

Other AKAPs that pair PKA with PDEs have been identified. PDE4D3 has been detected in the AKAP350 (AKAP450-Yotiao) complex at the centrosomal area of Sertoli cells [35], and other members of the PDE family have been shown to associate with AKAP complexes [21]. For example, in T cells, PDE4A is associated with several AKAPs, including AKAP95, AKAP149 and MTG16b, which has also been shown to target another PDE, PDE7A [21]. Although the functional implications of these interactions have not been fully investigated, the activity of these PDEs might be controlled by AKAPassociated kinases and phosphatases. Protein phosphatases, such as those found in AKAP350 and mAKAP complexes, might also contribute to regulation of local cAMP gradients by counterbalancing PKA phosphorylation events [36,37].

PDE activity can be regulated by other enzymes, such as members of the extracellular-signal-regulated kinase (ERK) family, that might also be present in these cAMPsensitive complexes $[19,38,39]$. Phosphorylation of long isoforms of PDE4 such as PDE4D3 by ERK2 inhibits activity and results in an increase level of intracellular
cAMP $[39,40]$. Therefore, an alternative feedback system might exist in which activation of the ERK-signaling pathway leads to inhibition of PDE activity and, hence, an increase in cAMP levels. This would, in turn, lead to PKA activation. Dodge-Kafka et al. [15] demonstrated that upstream stimulation of ERK leads to a sustained rather that a transient AKAP-PKA-PDE FRET signal. Furthermore, activation of ERK signaling in cardiomyocytes reduced the mAKAP-associated PDE activity [15]. Within the mAKAP complex, active ERK can suppress PDE activity, leading to an increase in anchored-PKA activity.

cAMP differentially regulates ERK signaling pathways in a manner that depends on cell type and growth condition [41-43]. In cultured rat neonatal ventriculocytes (RNVs), stimulation of cAMP synthesis prevented activation of ERK5 that is present in mAKAP complexes [15]. Inhibition of PKA did not reverse this effect, suggesting that a different cAMP effector was responsible. Epac1 co-precipitated with mAKAP from rat heart extract and was also present at the perinuclear membranes of hypertrophic RNVs, suggesting that it might be the cAMP effector preventing activation of ERK5 [15]. Biochemical data demonstrated that Epac1 is associated with the mAKAP complex by a direct interaction with PDE4D3. Furthermore, the Epac1-selective cAMP analog 8-CPT-2'$O$-Me-cAMP suppressed stimulation of mAKAP-associated ERK5 activity in RNVs $[15,44]$. Epac1 is a cAMPdependent guanine-nucleotide exchange factor (GEF) for the small RAS-like GTPase proteins repressor-activator proteins Rap1 and Rap2 [45-47]. Constitutively active RapGAP (a GTPase-activating protein that attenuates Rap1 activity) prevented the effect of cAMP on mAKAPassociated ERK5 activity, suggesting that Epac1 mediates its effects on ERK5 through Rap1 [15].

\section{Local cAMP levels and cardiac function}

Cardiac hypertrophy, an enlargement of heart-muscle cells, occurs in response to either exercise (physiological hypertrophy) or chronic hypertension (pathological hypertrophy) [48,49]. Physiological hypertrophy is beneficial because it enhances cardiac output to meet the increased metabolic demands of the body, whereas pathological hypertrophy is a major risk factor in the progression of heart failure [48,49]. A model for cardiac hypertrophy is provided by the treatment of RNVs with hypertrophic agonists. One such agent - leukemia inhibitory factor (LIF) - increases ERK5 activity in these cells [50]. MEK5, a dominant negative form of the activator of the MAPkinase ERK5, blocked LIF-induced 'eccentric hypertrophy', which is an increase in myofibril length. In addition, the expression of constitutively active MEK5 in the cardiac tissue of transgenic mice resulted in eccentric hypertrophy and heart failure [50]. Both inhibition of ERK-kinase activity and activation of Epac1 by 8-CPT-2'-O-Me-cAMP blocked the ability of LIF to increase the size of RNVs [15]. This was also partially blocked by PDE4 inhibition [15]. Clearly, the enzymes that make up the mAKAP complex have a role in facilitating LIF-induced hypertrophy. Indeed, knockdown by RNA interference (RNAi) of mAKAP suppressed the ability of LIF to induce hypertrophy in RNVs. This phenomenon was rescued when a 
form of mAKAP resistant to short hairpin RNA (shRNA) was expressed in the knockdown cells [15]. Furthermore, overexpression of a fragment of the mAKAP targeting sequence disrupted the perinuclear targeting of mAKAP and blocked the LIF-induced increase in cell size [15]. Thus, mAKAP targets cAMP-responsive enzymes to the perinuclear region of the cell to respond to upstream signals and modulate their effect on cardiomyocyte size.

The importance of local cAMP levels in cardiac function is also highlighted by a recent study in which PDE4Ddeficient mice have developed age-related cardiomyopathy, exercise-induced arrhythmias and heart failure [51]. Total cellular cAMP levels were similar in knockout and wildtype mice; however, there seemed to be differences within cellular microenvironments. In the Z-lines of cardiomyocytes from PDE4D-deficient mice, cAMP levels were increased compared with those observed in wild-type mice. PDE4D-deficient mice also showed enhanced PKA phosphorylation of the cardiac ryanodine receptor $\left(\mathrm{RY}_{2}\right.$ receptor), and these hyperphosphorylated, 'leaky' $R Y_{2}$ receptors exhibited defects in function. This finding is consistent with changes that were observed in human failing hearts. PDE4D3 is a component of a $\mathrm{RY}_{2}$ receptor complex in mouse and human cardiac tissue. Both levels of PDE4D3 bound to $R Y_{2}$ receptor and $\mathrm{RY}_{2}$ receptorassociated PDE4 activity were reduced to a similar degree in failing human and PDE4D-deficient mouse hearts. Importantly, there was an improvement in cardiac function that was observed in PDE4D-deficient mice in which the $\mathrm{RY}_{2}$ receptors could not be phosphorylated by PKA (RyR2-S2808A) [51]. Given that inhibitors of PDE4 are being developed as therapeutic agents for several chronic diseases, understanding the role of PDEs and regulation of local cAMP levels in the heart is essential. The role of distinct PDE isozymes in regulating local cAMP levels in neonatal rat cardiomyocytes was investigated using a cAMP biosensor that consists of $\mathrm{R}$ and $\mathrm{C}$ subunits of PKA that were labeled with fluorophores [7]. In this study, PDE3 and PDE4 were shown to have differential effects on resting cAMP levels and cAMP generated by the stimulation of $\beta$-adrenergic receptors. Furthermore, each PDE isoform was found in distinct subcellular locations in cultured cardiac myocytes, indicating that distinct pools of PDEs might have a role in the control of specific, localized pools of cAMP.

\section{Local cAMP levels and lipolysis}

Regulation of distinct pools of cAMP at specific sites within the cell is likely to be an important process in many other cell types. Historically, cAMP signaling was identified as a consequence of studies on lipolysis and insulin action. Additional understanding of the intricate balance of fuel storage and utilization in adipose-tissue metabolism suggests a probable role for AKAPs. Adipocytes are the main site of triglyceride storage in mammals [52,53]. Hormonal stimulation of $\beta$-adrenergic receptors on these cells increases cAMP levels and PKA activity, resulting in lipolysis of the stored triglycerides. PKA stimulates lipolysis by phosphorylating the hormone-sensitive lipase (HSL) and perilipin $[54,55]$. PKA also phosphorylates and activates PDE3B, reducing cAMP levels and, thereby, bringing PKA activity back to basal levels [56]. Insulin stimulation also activates PDE3B in a phosphatidylinositol-3-kinase (PI3-kinase)- and protein-kinase-B (PKB)-dependent manner, thereby reducing lipolysis $[53,57,58]$. Chronic insulin treatment of adipocytes, however, has been reported to increase isoproterenolinduced cAMP generation [59]. The effect of insulin treatment on cAMP-induced activation of PKA was measured using the PKA-activity reporter AKAR2 in 3T3-L1 adipocytes [4]. Chronic insulin treatment did not change basal AKAR2 phosphorylation, but the PKA response to isoproterenol was delayed. By contrast, there was no insulin-induced delay in PKA phosphorylation when cAMP levels were increased either by forskolin treatment or cAMP uncaging. This suggested that the insulin-induced delay was specific for a $\beta$-adrenergicassociated pool of PKA [4]. The RII $\beta$ subunit of PKA was associated with the $\beta$-adrenergic receptor in immunoprecipitates from 3T3-L1 adipocytes and maintained or slightly increased after isoproterenol treatment. Pretreatment of 3T3-L1 adipocytes with insulin, however, led to a reduction of this association. Importantly, use of the AKAP-PKA-anchoring inhibitor peptide, Ht31, suggested that the PKA is coupled to the $\beta$-adrenergic receptor through direct interaction with an AKAP [4]. In adipocytes, chronic hyperinsulinemia might weaken the association of an AKAP-anchored PKA to the $\beta$-adrenergic receptor, and activation of the receptor leads to disruption of the PKA- $\beta$-adrenergic-receptor complex, possibly through PKA phosphorylation mechanisms. AKAP79150 and Gravin are possible candidates to mediate the interaction with the $\beta$-adrenergic receptor [60-63].

\section{Concluding remarks}

AKAPs contribute to the specificity of cAMP signaling by recruiting PKA and other effector proteins to a given subcellular environment where the activation of precise enzyme combinations mediates the required biological outcome. Each effector has a distinct threshold for activation by cAMP. The mAKAP complex in the heart coordinates three enzymes: PKA, PDE4D3 and Epac1, to ensure the appropriate response at the local cAMP level as this ubiquitous second messenger is increased or decreased. Recently, it has been shown that a growing number of AKAPs also incorporate PDEs into the complex for fine-tuning of the cAMP gradient within a microdomain. This provides an additional level of sophistication that enables spatial and temporal control of cAMP flux.

Another potential function for these extended AKAP-PDE signaling complexes might be to suppress bursts of cAMP synthesis that emanate from receptor-Gprotein-adenylyl-cyclase complexes at the plasma membrane. This might be particularly relevant for the adenylyl cyclase isoforms AC5 and AC6 that are inhibited by PKA phosphorylation. [5,64,65]. If this latter configuration of signaling enzymes exists, it would provide an additional negative-feedback mechanism to synchronize the ebb and flow of cAMP as it diffuses across the cell.

Generation of the ubiquitous second messenger cAMP in response to many different extracellular stimuli requires tight regulation, but flexible, downstream 


\section{Box 1. Outstanding questions}

- What is the effective rate of cAMP diffusion in cells?

- Are there any AKAPs directly associated with adenylyl cyclases?

- Are there other classes of PDEs in AKAP complexes?

- What is the three-dimensional structure of an AKAP complex?

- Which roles do mAKAP signaling complexes have in the etiology of heart diseases?

machinery to ensure the appropriate cellular response. A growing body of work on the role that AKAPs have in the heart and in other tissues provides evidence that these multifunctional scaffolding proteins facilitate the fidelity of cAMP signaling. This tight control of cAMP signaling is clearly important in the maintenance of a healthy state, whereas the loss of this regulation might initiate diseases. Future translational studies will apply what we have learned about the cellular role of AKAPs to address the etiological questions of heart disease and other chronic illnesses (Box 1).

\section{Acknowledgements}

This work was supported by grant number DK54441 from the National Institutes of Health. We thank A. Goehring for critical reading of the manuscript.

\section{References}

1 Sutherland, E.W. (1972) Studies on the mechanism of hormone action. Science 177, 401-408

2 Tasken, K. and Aandahl, E.M. (2004) Localized effects of cAMP mediated by distinct routes of protein kinase A. Physiol. Rev. 84, $137-167$

3 Wong, W. and Scott, J.D. (2004) AKAP signalling complexes: focal points in space and time. Nat. Rev. Mol. Cell Biol. 5, 959-971

4 Zhang, J. et al. (2005) Insulin disrupts $\beta$-adrenergic signalling to protein kinase A in adipocytes. Nature 437, 569-573

5 Cooper, D.M. (2003) Regulation and organization of adenylyl cyclases and cAMP. Biochem. J. 375, 517-529

6 Hanoune, J. and Defer, N. (2001) Regulation and role of adenylyl cyclase isoforms. Annu. Rev. Pharmacol. Toxicol. 41, 145-174

7 Mongillo, M. et al. (2004) Fluorescence resonance energy transferbased analysis of cAMP dynamics in live neonatal rat cardiac myocytes reveals distinct functions of compartmentalized phosphodiesterases. Circ. Res. 95, 67-75

8 Rich, T.C. et al. (2000) Cyclic nucleotide-gated channels colocalize with adenylyl cyclase in regions of restricted cAMP diffusion. J. Gen. Physiol. 116, 147-161

9 Zaccolo, M. and Pozzan, T. (2002) Discrete microdomains with high concentration of cAMP in stimulated rat neonatal cardiac myocytes. Science 295, 1711-1715

10 Rich, T.C. et al. (2001) In vivo assessment of local phosphodiesterase activity using tailored cyclic nucleotide-gated channels as cAMP sensors. J. Gen. Physiol. 118, 63-78

11 Adams, S.R. et al. (1991) Fluorescence ratio imaging of cyclic AMP in single cells. Nature 349, 694-697

12 Zaccolo, M. et al. (2000) A genetically encoded, fluorescent indicator for cyclic AMP in living cells. Nat. Cell Biol. 2, 25-29

13 Zaccolo, M. and Pozzan, T. (2002) Discrete microdomains with high concentration of cAMP in stimulated rat neonatal cardiac myocytes. Science 295, 1711-1715

14 Zhang, J. et al. (2001) Genetically encoded reporters of protein kinase A activity reveal impact of substrate tethering. Proc. Natl. Acad. Sci. U. S. A. 98, 14997-15002

15 Dodge-Kafka, K.L. et al. (2005) The protein kinase A anchoring protein mAKAP coordinates two integrated cAMP effector pathways. Nature 437, 574-578

16 Chen, C. et al. (1999) Cyclic AMP diffusion coefficient in frog olfactory cilia. Biophys. J. 76, 2861-2867
17 Dworkin, M. and Keller, K.H. (1977) Solubility and diffusion coefficient of adenosine $3^{\prime}: 5^{\prime}$-monophosphate. J. Biol. Chem. 252, 864-865

18 Maurice, D.H. et al. (2003) Cyclic nucleotide phosphodiesterase activity, expression, and targeting in cells of the cardiovascular system. Mol. Pharmacol. 64, 533-546

19 Houslay, M.D. and Adams, D.R. (2003) PDE4 cAMP phosphodiesterases: modular enzymes that orchestrate signalling cross-talk, desensitization and compartmentalization. Biochem. J. 370, 1-18

20 Dodge, K.L. et al. (2001) mAKAP assembles a protein kinase A/PDE4 phosphodiesterase cAMP signaling module. EMBO J. 20, 1921-1930

21 Asirvatham, A.L. et al. (2004) A-kinase anchoring proteins interact with phosphodiesterases in T lymphocyte cell lines. J. Immunol. 173, 4806-4814

22 Dell'Acqua, M.L. and Scott, J.D. (1997) Protein kinase A anchoring. J. Biol. Chem. 272, 12881-12884

23 Newlon, M.G. et al. (1997) The A-kinase anchoring domain of type II $\alpha$ cAMP-dependent protein kinase is highly helical. J. Biol. Chem. 272, 23637-23644

24 Newlon, M.G. et al. (2001) A novel mechanism of PKA anchoring revealed by solution structures of anchoring complexes. EMBO J. 20, $1651-1662$

25 Angelo, R. and Rubin, C.S. (1998) Molecular characterization of an anchor protein $\left(\mathrm{AKAP}_{\mathrm{CE}}\right)$ that binds the RI subunit $\left(\mathrm{R}_{\mathrm{CE}}\right)$ of type $\mathrm{I}$ protein kinase A from Caenorhabditis elegans. J. Biol. Chem. 273, 14633-14643

26 Huang, L.J. et al. (1997) Identification of a novel dual specificity protein kinase A anchoring protein, D-AKAP1. J. Biol. Chem. 272, 8057-8064

27 Huang, L.J. et al. (1997) D-AKAP2, a novel protein kinase A anchoring protein with a putative RGS domain. Proc. Natl. Acad. Sci. U. S. A. 94, 11184-11189

28 Wang, L. et al. (2001) Cloning and mitochondrial localization of fulllength D-AKAP2, a protein kinase A anchoring protein. Proc. Natl. Acad. Sci. U. S. A. 98, 3220-3225

29 Dell'Acqua, M.L. et al. (1998) Membrane-targeting sequences on AKAP79 bind phosphatidylinositol-4, 5- bisphosphate. EMBO J. 17, 2246-2260

30 Trotter, K.W. et al. (1999) Alternative splicing regulates the subcellular localization of A-kinase anchoring protein 18 isoforms. J. Cell Biol. 147, 1481-1492

31 Sette, C. and Conti, M. (1996) Phosphorylation and activation of a cAMP-specific phosphodiesterase by the cAMP-dependent protein kinase. J. Biol. Chem. 271, 16526-16534

32 Carlisle Michel, J.J. et al. (2004) PKA phosphorylation of PDE4D3 facilitates recruitment of the mAKAP signaling complex. Biochem. J. $381,587-592$

33 Tao, J. et al. (2003) Protein kinase A regulates AKAP250 (gravin) scaffold binding to the $\beta 2$-adrenergic receptor. EMBO J. 22 , 6419-6429

34 Carnegie, G.K. et al. (2004) AKAP-Lbc nucleates a protein kinase D activation scaffold. Mol. Cell 15, 889-899

35 Tasken, K.A. et al. (2001) Phosphodiesterase 4D and protein kinase a type II constitute a signaling unit in the centrosomal area. J. Biol. Chem. 276, 21999-22002

36 Kapiloff, M.S. et al. (2001) mAKAP and the ryanodine receptor are part of a multi-component signaling complex on the cardiomyocyte nuclear envelope. J. Cell Sci. 114, 3167-3176

37 Westphal, R.S. et al. (1999) Regulation of NMDA receptors by an associated phosphatase-kinase signaling complex. Science 285, 93-96

38 Baillie, G. et al. (2001) Phorbol 12-myristate 13-acetate triggers the protein kinase A-mediated phosphorylation and activation of the PDE4D5 cAMP phosphodiesterase in human aortic smooth muscle cells through a route involving extracellular signal regulated kinase (ERK). Mol. Pharmacol. 60, 1100-1111

39 Hoffmann, R. et al. (1999) The MAP kinase ERK2 inhibits the cyclic AMP-specific phosphodiesterase HSPDE4D3 by phosphorylating it at Ser579. EMBO J. 18, 893-903

40 MacKenzie, S.J. et al. (2000) ERK2 mitogen-activated protein kinase binding, phosphorylation, and regulation of the PDE4D cAMP-specific phosphodiesterases. The involvement of $\mathrm{COOH}$-terminal docking sites and NH2-terminal UCR regions. J. Biol. Chem. 275, 16609-16617 
41 Cook, S.J. and McCormick, F. (1993) Inhibition by cAMP of rasdependent activation of raf. Science 262, 1069-1072

42 Vaillancourt, R.R. et al. (1994) B-Raf-dependent regulation of the MEK-1/mitogen-activated protein kinase pathway in PC12 cells and regulation by cyclic AMP. Mol. Cell. Biol. 14, 6522-6530

43 Pearson, G.W. and Cobb, M.H. (2002) Cell condition-dependent regulation of ERK5 by cAMP. J. Biol. Chem. 277, 48094-48098

44 Enserink, J.M. et al. (2002) A novel Epac-specific cAMP analogue demonstrates independent regulation of Rap1 and ERK. Nat. Cell Biol. 4, 901-906

45 Bos, J.L. (2003) Epac: a new cAMP target and new avenues in cAMP research. Nat. Rev. Mol. Cell Biol. 4, 733-738

46 de Rooij, J. et al. (1998) Epac is a Rap1 guanine-nucleotide-exchange factor directly activated by cyclic AMP. Nature 396, 474-477

47 Kawasaki, H. et al. (1998) A family of cAMP-binding proteins that directly activate Rap1. Science $282,2275-2279$

48 Olson, E.N. (2004) A decade of discoveries in cardiac biology. Nat. Med. $10,467-474$

49 Wakatsuki, T. et al. (2004) The biochemical response of the heart to hypertension and exercise. Trends Biochem. Sci. 29, 609-617

50 Nicol, R.L. et al. (2001) Activated MEK5 induces serial assembly of sarcomeres and eccentric cardiac hypertrophy. EMBO J. 20, $2757-2767$

51 Lehnart, S.E. et al. (2005) Phosphodiesterase 4D deficiency in the ryanodine-receptor complex promotes heart failure and arrhythmias. Cell 123, 25-35

52 Carmen, G.Y. and Victor, S.M. (2006) Signalling mechanisms regulating lipolysis. Cell. Signal. 18, 401-408

53 Holm, C. (2003) Molecular mechanisms regulating hormone-sensitive lipase and lipolysis. Biochem. Soc. Trans. 31, 1120-1124

54 Anthonsen, M.W. et al. (1998) Identification of novel phosphorylation sites in hormone-sensitive lipase that are phosphorylated in response to isoproterenol and govern activation properties in vitro. J. Biol. Chem. 273, 215-221

55 Zhang, H.H. et al. (2003) Lipase-selective functional domains of perilipin A differentially regulate constitutive and protein kinase A-stimulated lipolysis. J. Biol. Chem. 278, 51535-51542

56 Rahn, T. et al. (1996) Identification of the site in the cGMP-inhibited phosphodiesterase phosphorylated in adipocytes in response to insulin and isoproterenol. J. Biol. Chem. 271, 11575-11580

57 Kitamura, T. et al. (1999) Insulin-induced phosphorylation and activation of cyclic nucleotide phosphodiesterase 3B by the serinethreonine kinase Akt. Mol. Cell. Biol. 19, 6286-6296

58 Wijkander, J. et al. (1998) Insulin-induced phosphorylation and activation of phosphodiesterase $3 \mathrm{~B}$ in rat adipocytes: possible role for protein kinase B but not mitogen-activated protein kinase or p70 S6 kinase. Endocrinology 139, 219-227

59 Hupfeld, C.J. et al. (2003) $\beta$-Arrestin 1 down-regulation after insulin treatment is associated with supersensitization of $\beta 2$ adrenergic receptor $\mathrm{G} \alpha \mathrm{s}$ signaling in 3T3-L1 adipocytes. Proc. Natl. Acad. Sci. U. S. A. $100,161-166$

60 Fan, G. et al. (2001) The scaffold protein gravin (cAMP-dependent protein kinase-anchoring protein 250 ) binds the $\beta 2$-adrenergic receptor via the receptor cytoplasmic Arg-329 to Leu-413 domain and provides a mobile scaffold during desensitization. J. Biol. Chem. 276, 24005-24014

61 Fraser, I.D. et al. (2000) Assembly of an A kinase-anchoring protein$\beta(2)$-adrenergic receptor complex facilitates receptor phosphorylation and signaling. Curr. Biol. 10, 409-412

62 Shih, M. et al. (1999) Dynamic complexes of $\beta 2$-adrenergic receptors with protein kinases and phosphatases and the role of gravin. J. Biol. Chem. 274, 1588-1595

63 Tao, J. et al. (2003) Protein kinase A regulates AKAP250 (gravin) scaffold binding to the $\beta 2$-adrenergic receptor. EMBO J. 22, 6419-6429
64 Chen, Y. et al. (1997) Adenylyl cyclase 6 is selectively regulated by protein kinase A phosphorylation in a region involved in Gas stimulation. Proc. Natl. Acad. Sci. U. S. A. 94, 14100-14104

65 Premont, R.T. et al. (1992) Lowered responsiveness of the catalyst of adenylyl cyclase to stimulation by Gs in heterologous desensitization: a role for adenosine $3^{\prime}, 5^{\prime}$-monophosphate-dependent phosphorylation. Endocrinology 131, 2774-2784

66 Dodge, K.L. et al. (2001) mAKAP assembles a protein kinase A/PDE4 phosphodiesterase cAMP signaling module. EMBO J. 20, 1921-1930

67 Dodge-Kafta, K.L. et al. (2005) The protein kinase A anchoring protein mAKAP coordinates two integrated cAMP effector pathways. Nature 437, 574-578

68 Kapiloff, M.S. et al. (2001) mAKAP and the ryanodine receptor are part of a multi-component signaling complex on the cardiomyocyte nuclear envelope. J. Cell Sci. 114, 3167-3176

69 Kapiloff, M.S. et al. (1999) mAKAP: an A-kinase anchoring protein targeted to the nuclear membrane of differentiated myocytes. J. Cell Sci. 112, 2725-2736

70 Tasken, K.A. et al. (2001) Phosphodiesterase 4D and protein kinase a type II constitute a signaling unit in the centrosomal area. J. Biol. Chem. 276, 21999-22002

71 Schmidt, P.H. et al. (1999) AKAP350, a multiply spliced protein kinase A-anchoring protein associated with centrosomes. J. Biol. Chem. 274, 3055-3066

72 Sillibourne, J.E. et al. (2002) Centrosomal anchoring of the protein kinase CK1 $\delta$ mediated by attachment to the large, coiled-coil scaffolding protein CG-NAP/AKAP450. J. Mol. Biol. 322, 785-797

73 Coghlan, V.M. et al. (1994) Cloning and characterization of AKAP 95, a nuclear protein that associates with the regulatory subunit of type II cAMP-dependent protein kinase. J. Biol. Chem. 269, 7658-7665

74 Coghlan, V.M. et al. (1995) Association of protein kinase A and protein phosphatase 2B with a common anchoring protein. Science 267, 108-111

75 Collas, P. et al. (1999) The A-kinase-anchoring protein AKAP95 is a multivalent protein with a key role in chromatin condensation at mitosis. J. Cell Biol. 147, 1167-1180

76 Huang, L.J. et al. (1997) Identification of a novel protein kinase A anchoring protein that binds both type I and type II regulatory subunits. J. Biol. Chem. 272, 8057-8064

77 Steen, R.L. et al. (2000) Recruitment of protein phosphatase 1 to the nuclear envelope by A-kinase anchoring protein AKAP149 is a prerequisite for nuclear lamina assembly. J. Cell Biol. 150, 1251-1262

78 Nauert, J.B. et al. (1997) Gravin, an autoantigen recognized by serum from myasthenia gravis patients, is a kinase scaffold protein. Curr. Biol. 7, 52-62

79 Bregman, D.B. et al. (1991) Molecular characterization of bovine brain P75, a high affinity binding protein for the regulatory subunit of cAMP-dependent protein kinase II $\beta$. J. Biol. Chem. 266, 7207-7213

80 Carr, D.W. et al. (1992) Localization of the cAMP-dependent protein kinase to the postsynaptic densities by A-kinase anchoring proteins. Characterization of AKAP 79. J. Biol. Chem. 267, 16816-16823

81 Colledge, M. et al. (2000) Targeting of PKA to glutamate receptors through a MAGUK-AKAP complex. Neuron 27, 107-119

82 Gao, T. et al. (1997) cAMP-dependent regulation of cardiac L-type $\mathrm{Ca}^{2+}$ channels requires membrane targeting of PKA and phosphorylation of channel subunits. Neuron 19, 185-196

83 Hoshi, N. et al. (2003) AKAP150 signaling complex promotes suppression of the M-current by muscarinic agonists. Nat. Neurosci. $6,564-571$

84 Jo, I. et al. (2001) AQP2 is a substrate for endogenous PP2B activity within an inner medullary AKAP-signaling complex. Am. J. Physiol. Renal Physiol. 281, F958-F965

85 Klauck, T.M. et al. (1996) Coordination of three signaling enzymes by AKAP79, a mammalian scaffold protein. Science 271, 1589-1592 